\title{
Bevacizumab treatment of patients with breast cancer is associated with induction of MYH11.
}

Shahan Mamoor, MS 1

1shahanmamoor@gmail.com

East Islip, NY USA

Bevacizumab (Avastin) is an approved treatment option by the European Medicines Agency ${ }^{1}$ for more than a quarter billion women in the European Union, and despite having its indication withdrawn by the Food and Drug Administration in 2011 is still utilized in clinical trials in the United States ${ }^{2,3}$. We mined published microarray data ${ }^{4}$ from the PROMIX trial to understand in an unbiased fashion genes most transcriptionally perturbed by bevacizumab administration and how this interacted with a standard anthracycline and taxane chemotherapeutic regimen, epirubicin and docetaxel. We report here the induction of the myosin heavy chain MYH115 in the primary tumors of women with breast cancer treated with bevacizumab.

Keywords: breast cancer, MYH11, bevacizumab, Avastin, transcription factors in breast cancer, systems biology of breast cancer, targeted therapeutics in breast cancer. 
Breast cancer affects women worldwide with relatively high frequency ${ }^{6}$. Bevacizumab (Avastin), a monoclonal antibody targeted against the vascular endothelial growth factor A, VEGF-A, is utilized for the treatment of breast cancer worldwide7. Bevacizumab was formerly approved by the Food and Drug Administration in conjunction with paclitaxel for the treatment of metastatic breast cancer which had not previously been treated with chemotherapy for HER2-negative breast cancer; this indication was withdrawn on the basis that its full safety and efficacy had not been demonstrated ${ }^{2}$. For the over quarter of a billion women in the European Union, bevacizumab remains approved by the European Medicines Agency for the treatment of metastatic breast cancer ${ }^{1}$. To understand the transcriptional response to bevacizumab in an unbiased fashion at the transcriptome level, we mined published microarray data from $\mathrm{PROMIX}^{4}$, a phase II clinical trial measuring clinical responses to epirubicin, docetaxel and bevacizumab in women with breast cancer.

\section{Methods}

We used datasets GSE874554 and GSE7297010 for this global differential gene expression analysis in conjunction with GEO2R. GSE87455 was generated using Illumina HumanHT-12 V4.0 expression beadchip technology with $n=122$ primary breast tumors at baseline, $n=83$ primary tumors after Cycle 2 of treatment, consisting of only chemotherapy (epirubicin and docetaxel) and $n=70$ following addition of bevacizumab to treatment regimen in patients with breast cancer; analysis was performed using platform GPL10558. GSE72970 was generated using Affymetrix Human Genome U133 Plus 2.0 Array with $n=60$ tumors from patients with colorectal cancer receiving FOLFIRI chemotherapy treatment, and $n=22$ tumors from patients with colorectal cancer receiving FOLFIRI and bevacizumab; analysis was performed using platform GPL570. The Benjamini and Hochberg method of $p$-value adjustment was used for ranking of differential expression but raw $p$-values were used to assess statistical significance of global differential expression. Log-transformation of data was auto-detected, and the NCBI generated category of platform annotation was used. A statistical test was performed to evaluate whether MYH11 transcription factor gene expression was significantly different between primary breast tumors at baseline following chemotherapy (epirubicin and docetaxel) only and following both chemotherapy and bevacizumab in humans with breast cancer using a one way analysis of variance (ANOVA) with Dunnett's multiple comparisons test. A statistical test was performed to evaluate whether MYH11 transcription factor gene expression was significantly different between the tumors of patients with colorectal cancer receiving FOLFIRI or FOLFIRI and bevacizumab using a unpaired two-tailed t-test with Welch's correction. We used PRISM for all statistical analyses of differential gene expression in human breast cancer and colorectal cancer (Version 8.4.0)(455).

\section{Results}

We mined published microarray data 4,10 to understand in an unbiased fashion and at the systems level how transcription globally was most significantly altered in primary tumors from humans with cancer following treatment with the monoclonal antibody bevacizumab.

\section{Bevacizumab treatment results in transcriptional induction of MYH11 in the primary tumors of women with breast cancer.}

We analyzed data from the PROMIX trial ${ }^{4}$ wherein patients were treated for 6 cycles; each of the six cycles included treatment with epirubicin and docetaxel; bevacizumab was added to the treatment regimen only after cycle 2 . Tumor tissues were sampled at baseline, following administration of 2 cycles 
of epirubicin and docetaxel (prior to administration of bevacizumab), and after the addition of bevacizumab, at surgery. We identified the myosin heavy chain MYH11 as among the genes most differentially expressed in the primary tumors of human patients with breast cancer when comparing tumors at baseline, after 2 cycles of epirubicin and docetaxel, and at surgery, once bevacizumab had been administered for 4 cycles (Table 1). When sorting each of the genes expressed in the primary tumors of

\section{Bevacizumab treatment results in transcriptional induction of MYH11 in the tumors of patients with colorectal cancer.}

We turned to a second microarray dataset 10 to understand more thoroughly tissue-specific transcriptional modulation by bevacizumab in cancer, comparing global gene expression between the tumors of patients treated with FOLFIRI chemotherapeutic regimen or the tumors of patients treated with FOLFIRI and bevacizumab. We found MYH11 was among the genes most differentially expressed in the tumors of patients with colorectal cancer upon treatment with bevacizumab (Table 2). When sorting each of the genes expressed in primary colorectal tumors based on significance of change in expression between FOLFIRI-administered patients treated with bevacizumab or not, MYH11 ranked 5247 out of 54765 total transcripts (Table 2), equating to $90.4 \%$ differential expression. Differential expression of MYH11 in the primary tumors of bevacizumab-treated patients with colorectal cancer was statistically significant (Table $2 ; p=5.35 \mathrm{E}-03$ ).

\section{Discussion}

We provided evidence here that a protein of the cytoskeleton, myosin heavy chain MYH11 is among the genes whose expression changes most significantly in primary tumors of the breast and of the colon and rectum upon treatment with bevacizumab. Thus, a treatment whose direct molecular action is inhibition of a vascular endothelial growth factor to induce tumor regression in humans results in transcriptional induction of a cytoskeletal protein found as a fusion protein in acute myeloid leukemia 5 . 


\section{References}

1. Avastin. European Medicines Agency. https://www.ema.europa.eu/en/medicines/human/EPAR/ avastin.

2. Lenzer, J., 2011. FDA committee votes to withdraw bevacizumab for breast cancer.

3. Basho, R.K., Gilcrease, M., Murthy, R.K., Helgason, T., Karp, D.D., Meric-Bernstam, F., Hess, K.R., Herbrich, S.M., Valero, V., Albarracin, C. and Litton, J.K., 2017. Targeting the PI3K/AKT/mTOR pathway for the treatment of mesenchymal triple-negative breast cancer: evidence from a phase 1 trial of mTOR inhibition in combination with liposomal doxorubicin and bevacizumab. JAMA oncology, 3(4), pp.509-515.

4. Kimbung, Siker, Ida Markholm, Judith Bjöhle, Tobias Lekberg, Anna Von Wachenfeldt, Edward Azavedo, Ariel Saracco et al. "Assessment of early response biomarkers in relation to long-term survival in patients with HER2-negative breast cancer receiving neoadjuvant chemotherapy plus bevacizumab: results from the Phase II PROMIX trial." International journal of cancer 142, no. 3 (2018): 618-628.

5. Liu, P., Tarle, S.A., Hajra, A., Claxton, D.F., Marlton, P., Freedman, M., Siciliano, M.J. and Collins, F.S., 1993. Fusion between transcription factor CBF beta/PEBP2 beta and a myosin heavy chain in acute myeloid leukemia. Science, 261(5124), pp.1041-1044.

6. Bray, F., Ferlay, J., Soerjomataram, I., Siegel, R.L., Torre, L.A. and Jemal, A., 2018. Global cancer statistics 2018: GLOBOCAN estimates of incidence and mortality worldwide for 36 cancers in 185 countries. CA: a cancer journal for clinicians, 68(6), pp.394-424.

7. Ellis, L.M. and Kirkpatrick, P., 2005. Bevacizumab. Nature Reviews Drug Discovery, 4.

8. Del Rio, M., Mollevi, C., Bibeau, F., Vie, N., Selves, J., Emile, J.F., Roger, P., Gongora, C., Robert, J., Tubiana-Mathieu, N. and Ychou, M., 2017. Molecular subtypes of metastatic colorectal cancer are associated with patient response to irinotecan-based therapies. European Journal of Cancer, 76, pp. 68-75.

PAGE 4 
Table 1: Differential expression of MYH11 in the primary breast tumors of women treated with bevacizumab.

The rank of differential expression, probe ID, $p$-value with respect to global differential expression, Fstatistic, fold change in MYH11 transcription factor gene expression between primary tumors at baseline and primary tumors following administration of chemotherapy only ("E+D": epirubicin and docetaxel), fold change in MYH11 gene expression between primary tumors at baseline and primary tumors following addition of bevacizumab to epirubicin and docetaxel, gene and gene name are listed in this chart.

PAGE 5 
PAGE 6 


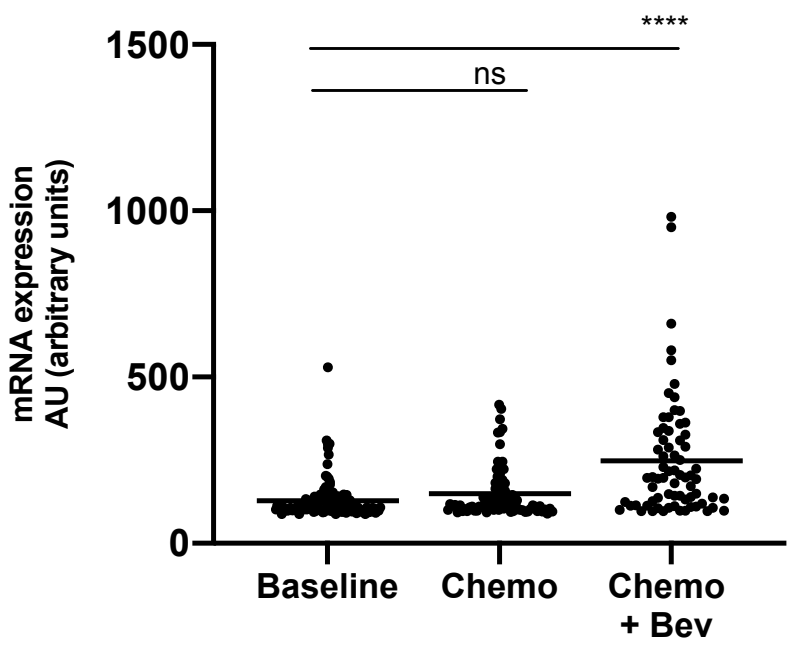

Figure 1: MYH11 is transcriptionally induced in the breast tumors of women following addition of bevacizumab to epirubicin and docetaxel treatment but not following treatment with epirubicin and docetaxel only.

Expression of MYH11 at the messenger RNA level from the tumors of patients enrolled in the PROMIX trial is graphically depicted here in primary tumors at baseline (left), in primary tumors following administration of 2 cycles of chemotherapy only (epirubicin and docetaxel) ("Chemo", middle), and in primary tumors at surgery, following addition of bevacizumab to treatment regimen ("Chemo + Bev", right), with mean MYH11 mRNA level marked and the result of a statistical test evaluating significance of difference in MYH11 expression between primary tumors of the breast and metastases to the brain in patients with breast cancer, a $\mathrm{p}$-value, listed above. $* * * *: p<0.0001$. ns: not significant; $p=0.2719$. 